\title{
Recent Advances in Colorectal Cancer Research: The Microenvironment Impact
}

\author{
Anne-Laure Pin • François Houle • Jacques Huot
}

Published online: 28 June 2011

(C) Springer Science+Business Media B.V. 2011

\section{Introduction}

Colorectal cancer (CRC) is the third most common cancer worldwide and represents $10 \%$ of all cancer-related deaths in North America. In many parts of the western world, CRC is the second leading cause of cancer deaths [1]. In most cases, death results as the consequence of the formation of liver metastases. In fact, hepatic metastases develop in 50\% of the patients and are responsible for two thirds of CRC patient deaths [2]. The genome of colon cancer cells is altered at several sites as a result of point mutations or changes in chromosome integrity [3]. The mutationassociated changes affect oncogenes, tumor suppressor genes and several metastasis-related genes such as cadherin and hypoxia-inducible factor-1 genes [4]. However, the dysregulation of these genes is not always a result of mutations. Other factors including epigenetic alterations such as $\mathrm{CpG}$ island hypermethylation, disruptions of histone modifiers and chromatin remodeling factors as well as deregulation of miRNA-mediated control of mRNA functions contribute to the incidence of cancer and metastasis [5]. Ten years ago, Hanahan and Weinberg have defined six phenotypic features by which genetic and epigenetic modifications interact to confer malignancy to solid cancers [3]: 1) Cancer cells have acquired growth selfsufficiency; 2) they are characterized by their insensitivity to anti-growth signals; 3 ) they have gained the capability to

\footnotetext{
A.-L. Pin $\cdot$ F. Houle $\cdot$ J. Huot $(\bowtie)$

Le Centre de recherche en cancérologie de l'Université Laval, l'Hôtel-Dieu de Québec, Centre de recherche du CHUQ, 9 rue McMahon,

Québec, Québec G1R 2J6 Canada

e-mail: Jacques.huot@fmed.ulaval.ca
}

escape apoptosis 4) they have lost the cell-autonomous program that limits their multiplication; 5) the growing neoplasm promotes angiogenesis and becomes capable of sustaining its oxygen and nutritive supplies; 6) cells from the primary neoplasm detach from this site and travel to distant sites that they will colonize to form metastases. These six features have since been considered as the essential hallmarks of cancer [3].

Recent studies have indicated that during the acquisition of genetic and epigenetic alterations that underlie the inherent hallmarks of cancer, transformed cells interact synergistically with their surrounding microenvironment [6]. In this context, the microenvironment influence on tumor progression, including the inflammatory response, is considered as a new essential feature of neoplastic transformation [7, 8]. Incidentally, Hanahan and Weinberg have recently revised their initial paper on "The hallmarks of cancer" to include the microenvironmental influence. Notably, they added avoiding immune destruction and dysregulating cellular energetics as emerging hallmarks of cancer [9]. Some of the key topics that characterize the insidious synergistic crosstalk between colon cancer and their microenvironment are reviewed in this special issue of Cancer Microenvironment.

\section{Diet as a Determinant of Colorectal Cancer Microenvironment}

A large proportion of CRCs are related to environmental factors. In particular, CRC is highly dependent on diet, which is a major modulator of cancer microenvironment. Notably, CRC is well recognized as being associated with obesity. In fact, several epidemiological studies have associated excess body weight with an increased risk of 
colon cancer, particularly for men $[10,11]$. In that regard, insulin resistance associated with obesity promotes colonocyte proliferation and suppresses apoptosis [12, 13]. Moreover, there is a strong association between meat consumption and colorectal cancer mortality $[14,15]$. The "meat-inducing effect" on CRC may be induced by mutagenic heterocyclic amines and polycyclic aromatic hydrocarbons that are formed during meat cooking at high temperatures. It can also result from nitrites and nitrite derivatives present in the processed meat products that are converted to carcinogenic $\mathrm{N}$-nitroso compounds in the colon [16]. High egg intake and high intake of total cholesterol are other dietetic risk factors of CRC [17]. In contrast, a long-term prospective study suggests that intake of fish and long-chain n-3 fatty acids from fish may decrease the risk of CRC [18].

Most interestingly, it has been reported that a high fat diet induces an hepatic steatosis, which establishes a permissive microenvironment for colorectal metastases [19]. Notably, signals including those elicited by TGF $\beta$ are initiated from steatotic host microenvironment and they set the proper conditions for tumor development even in the initial stage. As steatosis progresses, the susceptibility for tumor initiation increases with the accompanying inflammatory response. These findings are important because they clearly establish the link between diet, microenvironment and colon cancer metastasis. Along the same lines, Ann Chambers and her colleagues brought a series of arguments suggesting that diet can influence metastasis and tumor dormancy [20]. Notably, they report that genistein, a flavonoid found in soybean, may exert an anti-metastatic effect by influencing the interaction of metastatic cancer cells with their breast microenvironment [20,21]. Similarly, diet containing curcumin, an Indian spice, and the flavonoid quercetin appears to reduce the number and size of ileal and rectal adenomas in patients with familial adenomatous polyposis. Together, these findings suggest that foods containing high amounts of anticancer phytochemicals can be endowed with significant chemopreventive properties against CRC [22]. In this respect, regular drinking of green tea, a rich source of anticancer polyphenols (27) is associated with a $40 \%$ reduction in CRC risk in a cohort of 69,710 Chinese women (28). In CRC, chemoprevention has also been associated with dietary fibers, probiotics and prebiotics, vitamins $\mathrm{D}$ and $\mathrm{E}$, calcium and selenium, anthocyanins, phytoestrogens, epigallocatechin and resveratrol $[23$, 24]. The mechanisms underlying the chemopreventive action of these agents are multiple and involve the modulation of gene expression regulating cell proliferation, differentiation, apoptosis and the suppression of metastasis and angiogenesis [25]. Much remains to be done in this expending field that is addressed in Chapter 1 by Drs Denis Gingras and Richard Béliveau (Montreal, Canada).
Inflammation and Recruitment of Tumor-Associated Macrophages in Colorectal Cancer Microenvironment

The primary colon neoplasm contains several types of bone marrow-derived cells (BMDC) including tumor-associated macrophages (TAMs) $[26,27]$. The recruitment of TAMs to colon cancer is a typical response to cancer-associated inflammation and may explain why CRC responds to antiinflammatory agents [28]. It seems that TAMs favour CRC metastasis by secreting vascular endothelial growth factor (VEGF), thereby promoting angiogenesis at the primary site [29]. Moreover, TAMs trigger the removal of apoptotic CRC cells that express the sulfoglycolipids SM4s. This process is associated with an important phenotypic modification of TAMs that is characterized by an increased expression of TGF $\beta 1$ and secretion of IL- 6 . This phenotypic modification may contribute to further activate the angiogenic process [30]. Interestingly, neutrophils are also recruited to CRC and, as for TAMs, their recruitment is also associated with enhanced angiogenesis and metastasis [31]. On the other hand, several studies have reported the antitumor functions of TAM $[32,33]$. The possible anti-tumor effects of TAMs may rely on enhancing the nuclear translocation of macrophage migration inhibitory factor (MIF), and increasing the expression of GM-CSF and IL-12 [32]. Differentiation of TAMs to a cytotoxic macrophage subpopulation may also contribute to their antitumor functions [32]. Along these lines, infiltration of cancer masses by other BDMC such as CD8+ T cells and dendritic cells is associated with a favorable prognosis, as they destroy cancer cells [34]. Thus, it seems that TAMs and BDMC have either tumor-suppressive or tumor-promoting properties depending on the nature of $\mathrm{BDMC}$ and on the tissue context [35]. In the second chapter of the Issue, the role of TAMs in CRC development and progression has been reviewed by Drs Marco Erreni, Alberto Mantovani and Paola Allavena (Milan, Italy).

\section{miRNA are Major Regulators of Colorectal Cancer/ Microenvironment Interactions}

Hypoxia and angiogenesis are two important determinants by which cancer microenvironment influences CRC development and progression. Cancer cells cannot grow when they are located at a distance greater than $110 \mu \mathrm{m}$ from a blood vessel [36]. Accordingly, cancer cells begin to promote angiogenic neo-vascularization early in tumorigenesis in order to obtain oxygen and nutrients that will sustain their growth. At a later stage, angiogenesis is further involved since cancer cells that detach from the primary site use the new vessels as a path to reach and colonize new sites. Angiogenesis is thus importantly involved in CRC growth 
and dissemination. Notably, the level of angiogenesis is a survival predictor for CRC patients $[37,38]$. In accordance, patients having a Doppler vascularity index $>15 \%$ at the primary site have a poorer overall survival than patients with an index $<15 \%$ [38]. Most of the times, angiogenesis is switched on by tumor-associated hypoxic conditions, which contributes to activate the transcription of HIF-1. In turn, HIF-1 promotes the expression of angiogenic factors by the cancer cells including VEGF, bFGF (basic Fibroblast Growth Factor) and PLGF (Placental Derived-Growth Factor) [39]. Following hypoxia-induced gene expression by cancer cells, angiogenic factors interact with their receptors at the surface of endothelial cells initiating the activation of the angiogenic program. In particular, VEGF binds to VEGFR2, which triggers signaling cascades leading to the induction of vasodilation, endothelial cell migration/proliferation and vessel assembly [40]. The reciprocal synergistic interactions between cancer cells and endothelial cells constitute one of the best examples of the cancer cell/microenvironment interactions. As discussed in Chapter 2, pro-inflammatory cytokines (IL-6 and IL-1) released by stromal and cancer cells also mutually interact to favor tumor expansion and metastasis [41, 42].

microRNAs are single-stranded, evolutionarily conserved, small (17-22 ribonucleotides) non-coding RNAs. In the cells, miRNAs are generated from sequential processing of primary miRNA transcripts by the ribonuclease enzymes Drosha and Dicer [43]. These small RNAs regulate gene expression by binding to a target messenger RNA in its 3'UTR region, thereby repressing translation or inducing mRNA degradation. miRNA gene expression in CRC cells is regulated by the surrounding microenvironment via epigenetic mechanisms such as DNA methylation, histone acetylation or via enzymatic mechanisms that influence miRNA processing. The resulting dysregulation of miRNAs in the CRC cells will influence carcinogenesis if the mRNA targets are encoded by tumor suppressor genes or oncogenes. In this context, both overexpression and silencing of specific miRNAs may be involved in the carcinogenesis of CRC. Upregulation of mature miRNA may occur owing to transcriptional activation or amplification of the miRNA encoding gene, whereas silencing or reduced expression may result from deletion of a particular chromosomal region, epigenetic silencing, or defects in biogenesis. miRNAs regulate many known oncogenic and tumor suppressor pathways involved in the pathogenesis of CRC such as members of the Wnt/ $\beta$-catenin and phosphatidylinositol-3kinase (PI-3K) pathways, KRAS, p53, as well as regulators of the extracellular matrix or epithelial-mesenchymal transition [44]. Along these lines, several studies proposed that specific miRNAs may serve as markers of colorectal cancer detection and progression [43]. For example, high plasma level of miR-92 may be used to discriminate patients with colorectal and gastric cancers from normal healthy individuals [45].

Recent studies have revealed the important regulatory role played by miRNAs in regulating hypoxia and cooperating with VEGF in colon cancer progression [46]. In particular, the notion of an angioregulatory miRNA switch has been put forward [47]. According to this notion, the classical hypoxia switch involving HIF-1/cancer cells/ VEGF/endothelial cells axis is itself regulated by a tight balance between anti-angiogenic (miR-20a, miR-221) and pro-angiogenic (let-7f, miR-126) miRNAs [47].

The important emerging field of miRNA-mediated regulation of CRC progression, via regulation of pathways stimulated by VEGF and IL-6, is covered in Chapter 3 by Drs. Sabina Pucci and Paola Mazzarelli (Rome, Italy).

\section{Microenvironmental Control of Hepatic Metastasis Genes in Colorectal Cancer Cells}

Organ-specific colonization of cancer cells is a well-known characteristic of metastasis. For example, CRC metastasizes to liver and lungs, and intraocular cancer like uveal melanoma preferentially metastasizes to the liver but rarely to other organs [48]. This organ selectivity leads to the seed and soil hypothesis that was proposed by Stephen Paget more than 100 years ago [49]. According to this theory, the successful adhesive interactions of tumor cells (seeds) with the microenvironment of a particular organ (soil) are determinant for the formation of metastases in specific organs. Recent studies showed that the ability of colon cancer cells to interact and adhere with the liver microenvironment to implant and grow in this organ are influenced by the genetic, phenotypic and physiopathological backgrounds of the patients. In particular, three types of microenvironmentally-related genes are associated with the ability of colon cancer cells to form liver metastases in patients with advanced CRC: 1) Hepatic metastasis genes that are not expressed by tumor-unaffected liver tissue, 2) Genes co-expressed by hepatic metastases and tumorunaffected liver tissue, 3) Genes of tumor-unaffected liver tissue not expressed in hepatic metastases [50]. On the other hand, several experimental data highlight the point that the continous crosstalk between parenchymal and non parenchymal liver cells and the invading colon cancer cells favour metastasis [48]. For example, several lines of evidence indicate that hepatic ECM, by modulating the expression of growth factors and growth factor receptors in colon cancer cells, also modulates the proliferation of these cancer cells in the liver. Hepatocyte-derived ECM, in particular heparin proteoglycan, stimulates the proliferation of colon cancer cell lines via induction of autocrine growth factors and their receptors [6]. Additionnaly, the fact that 
cirrhosis may inhibit metastasis formation supports the view that the pathological background of the host liver also influences its colonization by colon cancer cells [51, 52].

Taken together, these findings clearly indicate that the selective formation of colon cancer metastasis to the liver is critically influenced by liver microenvironment. This fascinating field of research is reviewed by Dr. Fernando Vidal-Vanaclocha (Madrid, Spain) in the fourth chapter of the Issue.

\section{Colon Cancer Microenvironment Interactions in a Therapeutic Perspective}

From the topics described in this special issue of Cancer Microenvironment, it becomes clear that new therapeutic approaches will be developed based on interventions influencing the reciprocal colon cancer cell interactions with their microenvironment. In this context, the best example is the antitumoral effect exerted by antiangiogenic anti-VEGF agents such as bevacizumab/Avastin in expending up to 6 months the life span of CRC patients who are concomitantly treated with standard therapy such as FOLFOX (5-Fluorouracil, Folinic acid and Oxaliplatin) [53]. At the end of issue, Dr. Nicole Beauchemin (Montreal, Canada) summarizes the gains that can be expected in the next years by further exploiting the CRC microenvironment interactions towards therapeutic purposes.

\section{References}

1. Gellad ZF, Provenzale D (2010) Colorectal cancer: national and international perspective on the burden of disease and public health impact. Gastroenterology 138(6):2177-90.

2. Shimada H, Tanaka K, Endou I, et al (2009) Treatment for colorectal liver metastases: a review. Langenbecks Arch Surg 394(6):973-983

3. Hanahan D, Weinberg RA (2000) The hallmarks of cancer. Cell 100 (1):57-70

4. Lujambio A, Esteller M (2009) How epigenetics can explain human metastasis: A new role for microRNAs. Cell Cycle 8(3):377382

5. Esteller M (2008) Epigenetics in cancer. N Engl J Med 358 (11):1148-1159

6. Gout S, Huot J (2008) Role of cancer microenvironmentin metastasis: focus on colon cancer. Cancer Microenviron 1(1):69-83

7. Solinas G, Marchesi F, Garlanda C et al (2010) Inflammationmediated promotion of invasion and metastasis. Cancer Metastasis Rev 29(2):243-248

8. Colotta F, Allavena P, Sica A et al (2009) Cancer-related inflammation, the seventh hallmark of cancer: links to genetic instability. Carcinogenesis 30(7):1073-1081

9. Hanahan D, Weinberg RA (2011) Hallmarks of cancer: the next generation. Cell 144(5):646-674

10. Ning Y, Wang L, Giovannucci EL (2010) A quantitative analysis of body mass index and colorectal cancer: findings from 56 observational studies. Obes Rev 11(1):19-30
11. Moghaddam AA, Woodward M, Huxley R (2007) Obesity and risk of colorectal cancer: a meta-analysis of 31 studies with 70,000 events. Cancer Epidemiol Biomarkers Prev 16(12):2533-2547

12. Vanamala J, Tarver CC, Murano PS (2008) Obesity-enhanced colon cancer: functional food compounds and their mechanisms of action. Curr Cancer Drug Targets 8(7):611-633

13. Murthy NS, Mukherjee S, Ray G et al (2009) Dietary factors and cancer chemoprevention: an overview of obesity-related malignancies. J Postgrad Med 55(1):45-54

14. Khan N, Afaq F, Mukhtar H (2010) Lifestyle as risk factor for cancer: Evidence from human studies. Cancer Lett 293(2):133-143

15. Bingham SA, Pignatelli B, Pollock JR et al (1996) Does increased endogenous formation of $\mathrm{N}$-nitroso compounds in the human colon explain the association between red meat and colon cancer? Carcinogenesis 17(3):515-523

16. Oba S, Shimizu N, Nagata C et al (2006) The relationship between the consumption of meat, fat, and coffee and the risk of colon cancer: a prospective study in Japan. Cancer Lett 244 (2):260-267

17. Chao A, Thun MJ, Connell CJ et al (2005) Meat consumption and risk of colorectal cancer. JAMA 293(2):172-182

18. Hall MN, Chavarro JE, Lee IM et al (2008) A 22-year prospective study of fish, n-3 fatty acid intake, and colorectal cancer risk in men. Cancer Epidemiol Biomarkers Prev 17(5):1136-1143

19. VanSaun MN, Lee IK, Washington MK et al (2009) High fat diet induced hepatic steatosis establishes a permissive microenvironment for colorectal metastases and promotes primary dysplasia in a murine model. Am J Pathol 175(1):355-364

20. Chambers AF (2009) Influence of diet on metastasis and tumor dormancy. Clin Exp Metastasis 26(1):61-66

21. Vantyghem SA, Wilson SM, Postenka CO et al (2005) Dietary genistein reduces metastasis in a postsurgical orthotopic breast cancer model. Cancer Res 65(8):3396-3403

22. Cruz-Correa M, Shoskes DA, Sanchez P et al (2006) Combination treatment with curcumin and quercetin of adenomas in familial adenomatous polyposis. Clin Gastroenterol Hepatol 4(8):1035-1038

23. Demeule M, Michaud-Levesque J, Annabi B et al (2002) Green tea catechins as novel antitumor and antiangiogenic compounds. Curr Med Chem Anticancer Agents 2(4):441-463

24. Yang G, Shu XO, Li H et al (2007) Prospective cohort study of green tea consumption and colorectal cancer risk in women. Cancer Epidemiol Biomarkers Prev 16(6):1219-1223

25. Olejnik A, Tomczyk J, Kowalska K, et al (2010) The role of natural dietary compounds in colorectal cancer chemoprevention. Postepy Hig Med Dosw (Online) 64:175-187

26. Condeelis J, Pollard JW (2006) Macrophages: obligate partners for tumor cell migration, invasion, and metastasis. Cell 124 (2):263-266

27. Pollard JW (2004) Tumour-educated macrophages promote tumour progression and metastasis. Nat Rev Cancer 4(1):71-78

28. Mantovani A, Allavena P, Sica A et al (2008) Cancer-related inflammation. Nature 454(7203):436-444

29. Barbera-Guillem E, Nyhus JK, Wolford CC et al (2002) Vascular endothelial growth factor secretion by tumor-infiltrating macrophages essentially supports tumor angiogenesis, and IgG immune complexes potentiate the process. Cancer Res 62(23):7042-7049

30. Popovic ZV, Sandhoff R, Sijmonsma TP et al (2007) Sulfated glycosphingolipid as mediator of phagocytosis: SM4s enhances apoptotic cell clearance and modulates macrophage activity. J Immunol 179(10):6770-6782

31. Murdoch C, Muthana M, Coffelt SB et al (2008) The role of myeloid cells in the promotion of tumour angiogenesis. Nat Rev Cancer 8(8):618-631

32. Shih JY, Yuan A, Chen JJ-W et al (2006) Tumor-associated macrophage: its role in cancer invasion and metastasis. J Cancer Mol 2:101-106 
33. Mantovani A, Bottazzi B, Colotta F et al (1992) The origin and function of tumor-associated macrophages. Immunol Today 13 (7):265-270

34. Talmadge JE, Donkor M, Scholar E (2007) Inflammatory cell infiltration of tumors: Jekyll or Hyde. Cancer Metastasis Rev 26 (3-4):373-400

35. Joyce JA, Pollard JW (2009) Microenvironmental regulation of metastasis. Nat Rev Cancer 9(4):239-252

36. Kerbel R, Folkman J (2002) Clinical translation of angiogenesis inhibitors. Nat Rev Cancer 2(10):727-739

37. Goh V, Padhani AR, Rasheed S (2007) Functional imaging of colorectal cancer angiogenesis. Lancet Oncol 8(3):245-255

38. Chen CN, Cheng YM, Liang JT et al (2000) Color Doppler vascularity index can predict distant metastasis and survival in colon cancer patients. Cancer Res 60(11):2892-2897

39. Liao D, Johnson RS (2007) Hypoxia: a key regulator of angiogenesis in cancer. Cancer Metastasis Rev 26(2):281-290

40. Lamalice L, Le Boeuf F, Huot J (2007) Endothelial cell migration during angiogenesis. Circ Res 100(6):782-794

41. Lewis AM, Varghese S, Xu H et al (2006) Interleukin-1 and cancer progression: the emerging role of interleukin-1 receptor antagonist as a novel therapeutic agent in cancer treatment. J Transl Med 4:48

42. Scheller J, Ohnesorge N, Rose-John S (2006) Interleukin-6 transsignalling in chronic inflammation and cancer. Scand J Immunol 63(5):321-329

43. Aslam MI, Taylor K, Pringle JH et al (2009) MicroRNAs are novel biomarkers of colorectal cancer. Br J Surg 96(7):702-710
44. Slaby O, Svoboda M, Michalek J et al (2009) MicroRNAs in colorectal cancer: translation of molecular biology into clinical application. Mol Cancer 8:102

45. Spizzo R, Nicoloso MS, Croce CM et al (2009) SnapShot: MicroRNAs in Cancer. Cell 137(3):586-e1

46. Kulshreshtha R, Davuluri RV, Calin GA et al (2008) A microRNA component of the hypoxic response. Cell Death Differ 15(4):667671

47. Heusschen R, van Gink M, Griffioen AW et al (2010) MicroRNAs in the tumor endothelium: novel controls on the angioregulatory switchboard. Biochim Biophys Acta 1805 (1):87-96

48. Gout S, Tremblay PL, Huot J (2008) Selectins and selectin ligands in extravasation of cancer cells and organ selectivity of metastasis. Clin Exp Metastasis 25(4):335-344

49. Paget D (1889) The distribution of secondary growths in cancer of the breast. Lancet 1:571-573

50. Vidal-Vanaclocha F (2008) The prometastatic microenvironment of the liver. Cancer Microenviron 1(1):113-129

51. Seymour K, Charnley RM (1999) Evidence that metastasis is less common in cirrhotic than normal liver: a systematic review of post-mortem case-control studies. Br J Surg 86(10):12371242

52. Uetsuji S, Yamamura M, Yamamichi K et al (1992) Absence of colorectal cancer metastasis to the cirrhotic liver. Am J Surg 164 (2):176-177

53. Ellis LM, Haller DG (2008) Bevacizumab beyond progression: does this make sense? J Clin Oncol 26(33):5313-5315 